\title{
Hyper-finger for Remote Minimally Invasive Surgery in Deep Area
}

\author{
Koji Ikuta, Shinichi Daifu, Takahiko Hasegawa, and Humihiro Higashikawa \\ Department of Micro System Engineering, Graduate School of Engineering, Nagoya University \\ Furo-cho, Chikusa-ku, Nagoya, Aichi, 464-8603, Japan \\ Tel: +81 52-789-5024, Fax: +81 52-789-5027, \\ ikuta@mech.nagoya-u.ac.jp
}

\begin{abstract}
A new robotic system named Hyper Finger for minimally invasive surgery in deep organs has been developed. The finger size of the latest version is $10 \mathrm{~mm}$ and the entire system is much smaller and lighter, and can be set up on a camera tripod. This is one of the smallest master-slave robots in medicine. Each finger has nine degrees of freedom and several unique mechanisms are employed to solve the fundamental issues of conventional wire drive manipulators. The new concept and system were verified successfully by in-vivo remote minimally invasive surgery. Further improvements of the system toward a clinical version are now underway.
\end{abstract}

\section{Introduction}

A major focus of advanced medical technology development based on mechatronics is minimally invasive techniques in surgical operations, particularly the development of surgical instruments for laparoscopic surgery. We have been proposing and developing a Hyper Finger (hyper redundant multiple degrees of freedom active forceps) as a next-generation surgical instrument that enables more advanced and extensive laparoscopic surgery [1][2].

This study describes miniaturization of the arm to $10 \mathrm{~mm}$ diameter for clinical use, development of a compensation mechanism for drive wire elongation, and construction of a master-slave system with a pair of Hyper Fingers with this arm and mechanism.

Finally, further miniaturized prototype of Hyper Finger with nine degree of freedom was produced. The effectiveness of the system was also verified by in-vivo experiments.

\section{Remote Laparoscopic Surgery Using Hyper Fingers}

Different from standard open abdominal surgery, laparoscopic surgery is a minimally invasive operation technique, performed in the abdominal cavity with surgical instruments inserted through skin incisions of about $10 \mathrm{~mm}$ near the affected area. This 
surgical method is performed in the abdominal cavity which is expanded like a dome by gas pressure, using inserted instruments such as an endoscope to look inside the cavity, working forceps, etc. Compared to open abdominal surgery, this surgical approach offers the advantage of less physical and mental stress for patients due to the smallness of the incisions. However, the approach is still under development and entails the following problems.

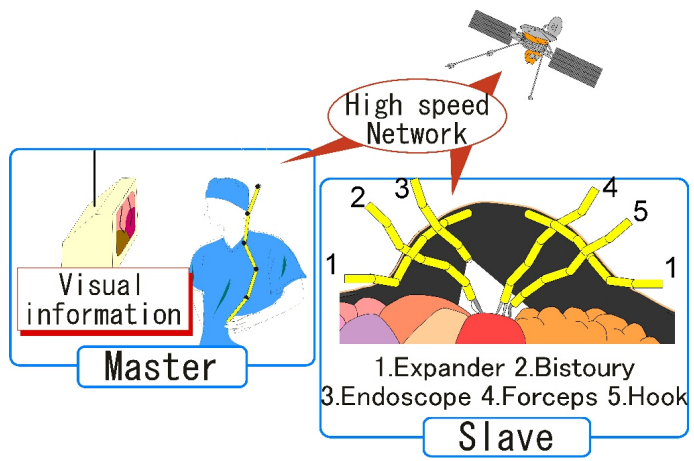

Fig. 1. Schematic diagram of remote laparoscopic surgery by using several type of Hyper Finger

1) Difficulty of observation and operation on a deep organ or on the backside of an organ

2) Limited operation area due to straight shaped forceps

3) Difficulty in cooperative work by many doctors

We have been developing the Hyper Finger (hyper redundant multiple degrees of freedom active forceps) surgical instrument to solve these problems [1].

As shown in Fig. 1, Hyper Fingers are active forceps with redundant degree-offreedom multiple joints, and various functions can be attained by exchanging endeffectors. Independent or cooperative operation of two or more Hyper Fingers with high degrees of freedom enables advanced and extensive laparoscopic surgery to be performed.

This method can be developed for remote laparoscopic surgery with a master-slave system through high-speed communication of the endoscopic image, positional information and power sensor information of Hyper Fingers. A person at a remote location or under radiation exposure can thus receive advanced laparoscopic surgery.

\section{Functional Verification Model}

In our previous study, we developed a master-slave system with a pair of Hyper Fingers (Fig. 2) for feasibility check.

Although the system is wire driven, decoupled drive is possible in all degrees of freedom due to the use of two new mechanisms: an active universal joint and a de- 
coupled link mechanism. The results showed good performance in position tracking and responsiveness of the master-slave system as well as enough force for surgery [2].

However, it was impossible to use this prototype for laparoscopic surgery because it was fabricated as a functional verification model of twice the size of devices used for surgery, as well as the following problems in clinical application:

1) Model of twice the size of devices used for surgery

2) Wire elongation

3) Friction between wire and wire path

4) Development of surgical end-effectors

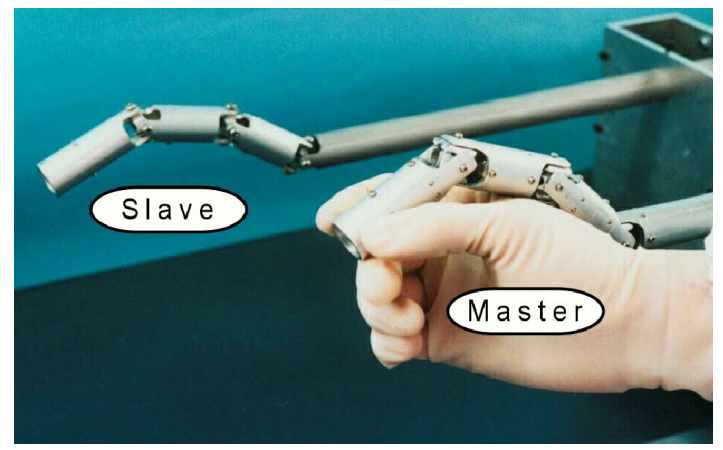

Fig. 2. First prototype of Master-Slave Hyper Finger (Mark-1 with 6 D.O.F.) [2]

\section{Development of a Surgically Applicable Prototype}

Figure 3 shows the developed miniaturized prototype of Hyper Finger (Mark-2) which is applicable to laparoscopic surgery. This model is not only smaller with an outer diameter of just $10 \mathrm{~mm}$, but also has new movement and arm mechanisms to solve the above problems. Better position tracking, stability and expandability were thus achieved compared with the previous functional verification model. The new mechanisms are as follows:

1) Decoupled ring joint

2) Compensation mechanism for wire elongation

3) Unit-type regular hexagonal pyramid structure

4) Detachable gripper mechanism

\subsection{Decoupled Ring Joint}

The decoupled ring joint consists of a pair of arms connected to each other with a joint ring, and allows two degrees of freedom (horizontal/vertical) rotation manipulated by two wires each (Fig. 4). Since each axis and the corresponding wireconnecting points are located in the same plane, one rotational drive system has no effect on the other. Thus, each joint can be driven independently with two degrees of freedom. 

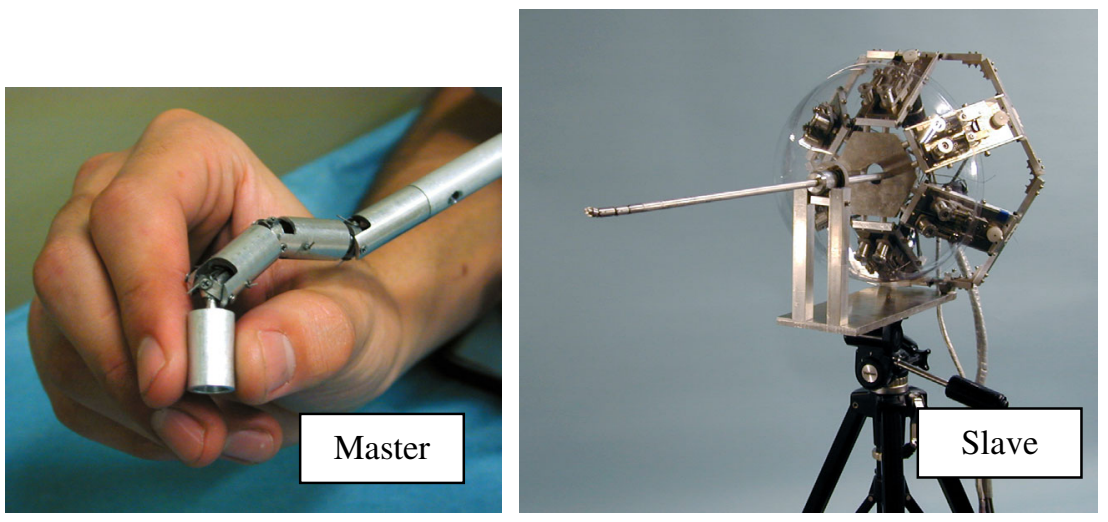

Fig. 3. Miniaturized Master-Slave Hyper Finger in real size (Mark-2 with 7 D.O.F.)

In addition, the friction loss is very small due to no wire folding at each joint. Therefore, there are no differences in drive characteristics between vertical and horizontal directions, and so this new joint system provides smoother drive compared with the active universal joint described in our previous reports.

Owing to its simple structure, the diameter is successfully reduced to $10 \mathrm{~mm}$, which is sufficiently small for laparoscopic surgery.

\subsection{Compensation Mechanism for Wire Elongation}

In a wire drive system, repeated stress generally causes wire elongation, which reduces the accuracy of positioning. The new mechanism devised by Hirose et al., which compensates for the wire elongation, was adopted to solve the problem [3].

Figure 6 shows the scheme of the compensation mechanism for wire elongation. When the wire is under high tension during driving, the static friction between the friction bar and the wire moves the pedestal so that the route length of the wire is kept constant. At the same time, the tension pulley and the coil springs stay still on the pedestal and do not affect driving. On the contrary, when the wire elongates to reduce the tension, the friction with the friction bar decreases, the spring rolls up the elongated wire and thus the mechanism keeps the route length constant. This mechanism improves the positioning accuracy and ensures long-time stable driving.

\subsection{Unit-Type Regular Hexagonal Pyramid Structure}

A drive mechanism having one degree of freedom consists of a motor, a potentiometer and a elongated wire compensation mechanism. Six units were configured to create a regular hexagonal pyramid. The completed module is shown in Fig. 3.

Since the six units are attached to the hexagonal pyramid frame, the whole module is very light and can be mounted on a basic camera tripod. Robots must be small to fit in operating rooms which are packed with various precision machines; there is no small, light surgery robot system that can rival our system. 
Moreover, the friction loss is very small due to the direct wire insertion from each unit to the arm, which requires no wire folding, and also the number of degrees of freedom can be easily increased thanks to the unit-type structure.

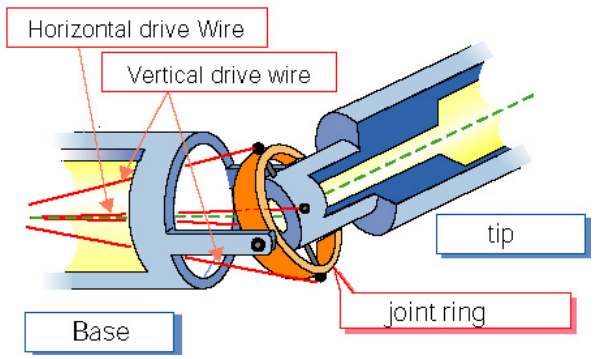

Fig. 4. Schematic design of active ring joint driven by decoupled wire drive mechanism
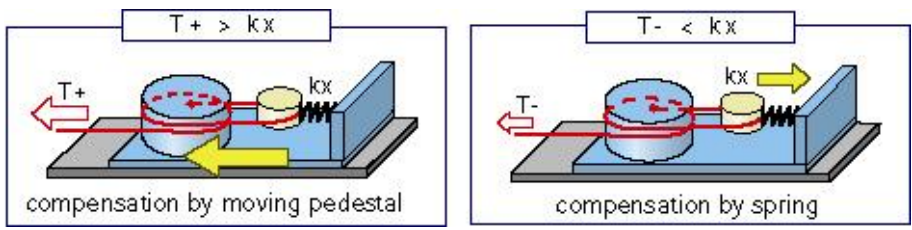

Fig. 6. Schematic design of compensating elongated wire mechanism

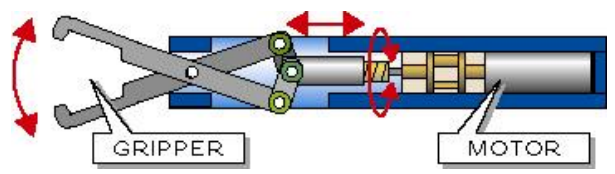

Fig. 7. (a) Schematic design of gripper mechanism installable on Hyper Finger

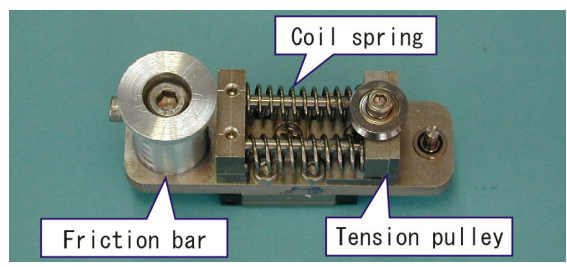

Fig. 5. Compensating elongated wire mechanism 
Using a link mechanism, the gripper can widely open up to about 40 degrees. Furthermore, the screw mechanism of the gripper gives the gripper sufficient holding power to maintain posture and force, to reduce errors such as slipping off an organ.

\section{Control System}

The block diagram of the control system is shown in Fig. 8. The system consists of a master Hyper Finger controlled by the doctor, a slave Hyper Finger inserted in the abdominal cavity for operation, and a control PC. The master has as many potentiometers to read the arm angles as degrees of freedom. The slave has as many potentiometers, drive motors and their motor drivers as degrees of freedom.

Backlash-free drive is possible at the master, and by manipulating each joint of the master, the corresponding slave joint follows the master.

Unilateral control, the most simple master-slave control system, is adopted for the present control system. By mounting force sensors, the system can easily be evolved to a bilateral control system in the future. Both the master and the slave adopt decoupled wire drive mechanisms, so there is no need for complicated calculations.

\section{Driving Experiments}

A position tracking experiment was conducted with this master-slave system. Fig.9 shows the position tracking result of the slave, which follows the master. The master was manually made to follow a sine wave with $\pm 40 \mathrm{deg}$. joint amplitude per cycle of about 10seconds. From viewpoint of response and deviation, all joints showed excellent tracking characteristics, even though the worst delay was $0.4 \mathrm{sec}$ in base joint.

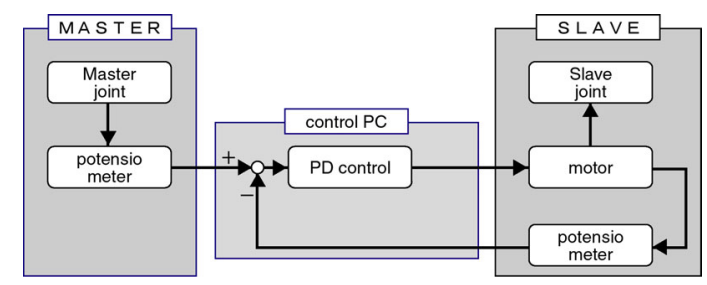

Fig. 8. Total control system for master-slave Hyper Finger
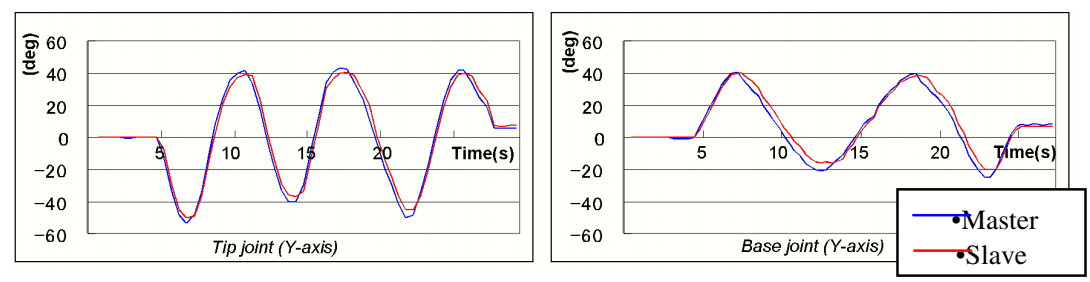

Fig. 9. Master-slave control of each active joint 


\section{In-vivo Experiment by Surgeons}

The effectiveness of the surgical Hyper Finger with the new mechanism was tested on an animal. The experiment was conducted using an anesthetized pig by the lifting method under nearly the same conditions as practical laparoscopic surgery. In the experiment, the doctor manipulated the master while watching the endoscopic image on the monitor and conducted slave operations in the abdominal cavity such as lifting an organ. Figure 10 shows the experiment in progress.

The results and discussion are summarized below.

The Hyper Finger had no problems in term of movement area and drive speed. The Hyper Finger was also able to lift the liver to secure space for surgery with the gripper mechanism on the end-effector, thus verifying that the holding force of the Hyper Finger is sufficient for surgery. Moreover, the Hyper Finger could be attached to a camera tripod, enabling it to be placed anywhere and thus avoid interference with other surgical instruments and any spatial problems.

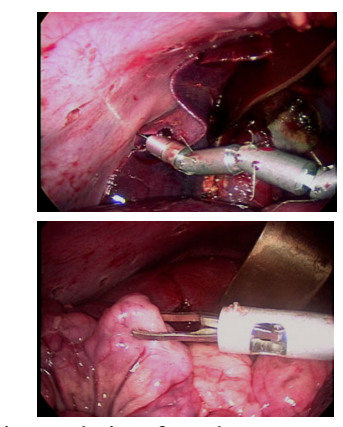

internal view from laparoscope

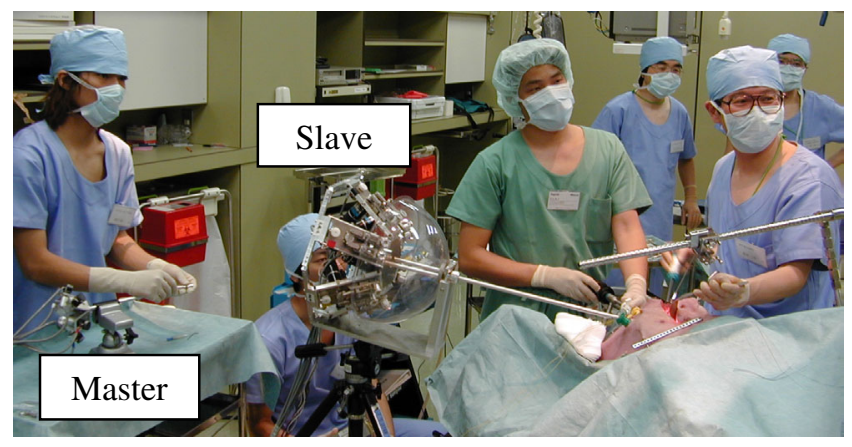

Fig. 10. In vivo surgical experiments with anesthetized pig

\section{New Hyper-finger (Mark-3) for Clinical Use}

A further miniaturized version with higher degree of freedom of hyper finger for clinical testing was developed. This model has the following new functions suitable for minimally invasive operation in deep sites.

1) Nine degrees of freedom

2) Rotating gripper with wire drive

3) Detachable finger mechanism

4) Small and light weight suitable for remote surgery by plural doctors

These additional new functions were verified through in-vivo experiment by doctors. All of the unique mechanisms verified by Hyper Finger (Mark 2) were introduced in the new version.

Figure 11 shows the clinical size Master-slave New Hyper Finger (Mark 3). Both the master and slave have been made far smaller. The diameter of the slave finger is 
$10 \mathrm{~mm}$ and it is sufficiently light to be set on a camera tripod. Several types of endeffector have been developed and can easily be replaced during surgery because each finger is detachable as shown in Fig. 12.

Figure 13 shows the new driving mechanism of the wire drive gripper satisfying rotational operation with enough torque. , Since rotation of only a tip part is possible, this gripper makes it much easier to perform a suture in a deep site.

Figure 14 shows the surgical experiment using the miniaturized Hyper Finger (Mark-3). The doctor could maneuver the master finger with the same nine degrees of freedom. Suturing of a deep organ was done without much difficulty. According to the doctor's opinion after remote operation of the pig, the force and torque were sufficiently high and the feeling of maneuvering was very smooth.

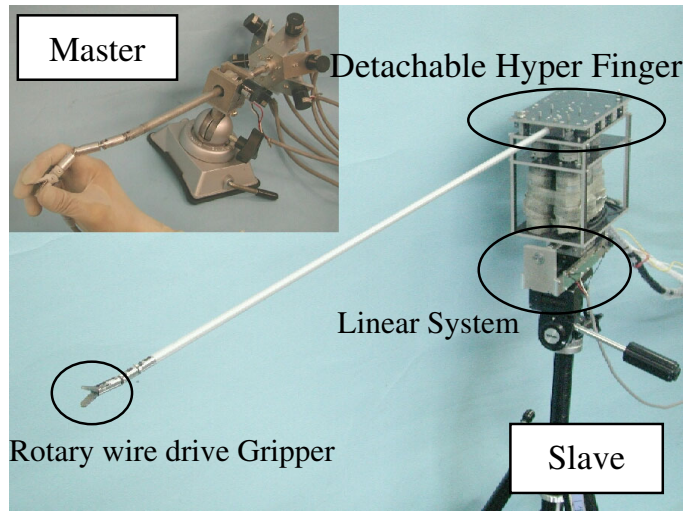

Fig. 11. Clinical size prototype Hyper Finger (Mark-3 with 9 D.O.F) set on camera tripod

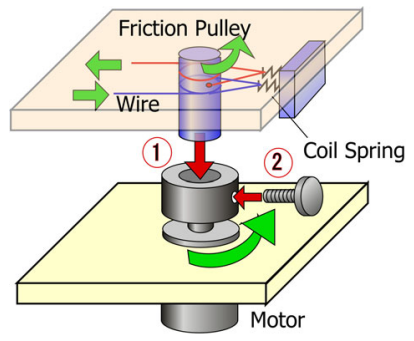

Fig. 12. Drive mechanism of Detachable Hvper Finger
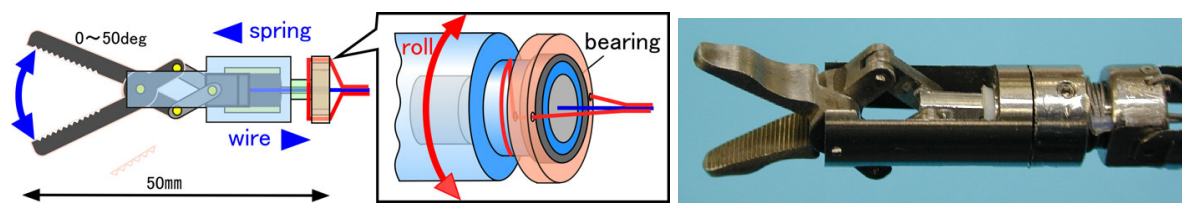

Fig. 13. Rotary wire drive Gripper

\section{Conclusions}

A new robotic system named Hyper Finger for minimally invasive surgery in deep organs has been developed. The finger size of the latest version is $10 \mathrm{~mm}$ and the entire system is much smaller and lighter, and can be set up on a camera tripod. This is one of the smallest master-slave robots in medicine. Each finger has nine degrees of freedom and several unique mechanisms are employed to solve the fundamental issues of conventional wire drive manipulators. The new concept and system were verified successfully by in-vivo remote minimally invasive surgery. Further improvements of the system toward a clinical version are now underway. 

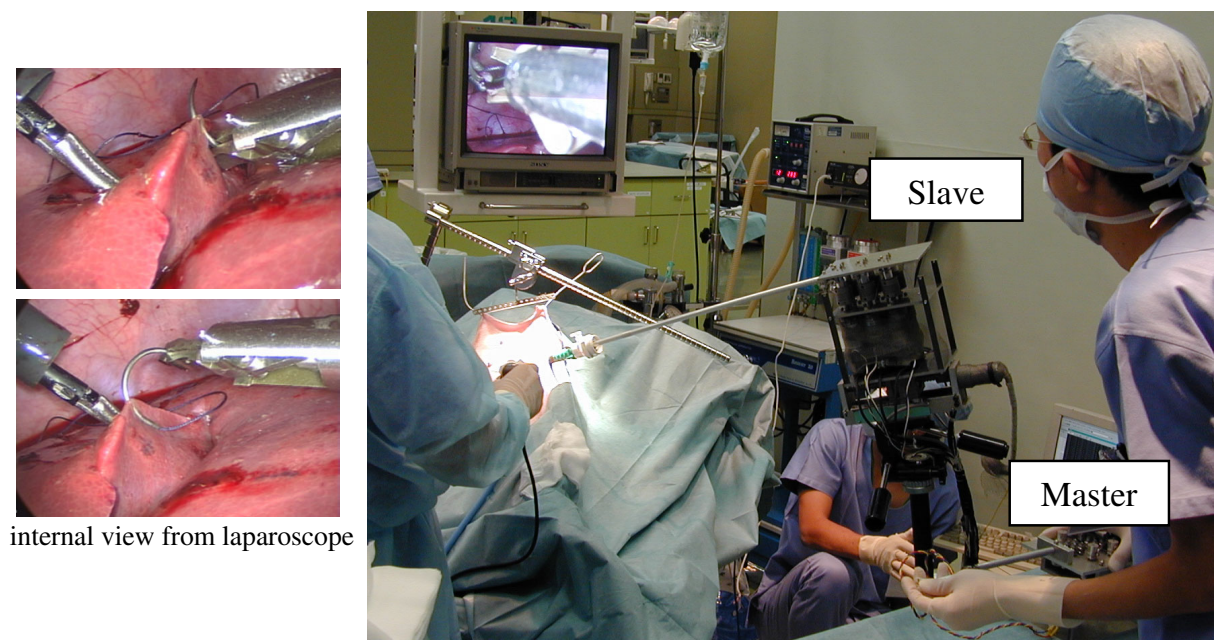

Fig. 14. In-vivo surgical experiments using clinical sized Hyper Finger Mark-3

\section{Acknowledgments}

The authors thank Dr. Daijou Hashimoto and Dr. Kazuhiro Shinohara at Saitama Medical School for their assistance in the animal experiment. We also acknowledge the assistance of Shinko Senda at Nagoya University and all contributors.

\section{References}

1. K.Ikuta, M.Nokata, S.Aritomi, "BIOMEDICAL MICRO ROBOTS DRIVEN BY MINIATURE CYBERNETIC ACTUATOR", Proc. of International Workshop on Micro Electromechanical Systems (MEMS'94), pp263-268, 1994

2. K.Ikuta, F.Higashikawa, K.Ogata "Study on Hyper Finger for Remote Minimal Invasive Surgery" Plenary Proc. of 1999 JSME Conference on Robotics and Mechatronics (ROBOMEC 99),1P2-10-005,1999

3. Shigeo Hirose, Richard Chu; Development of a light weight Torque Limiting M-Drive Actuator for Hyper Redundant Manipulator Float Arm, To be appeared in Proc.IEEE Int.on Robotics and Automation, 2831-2836,1999 